\title{
Interventioneller Verschluss eines offenen Foramen ovale reduziert das Rezidivrisiko
}

Fragestellung: Ist bei Patienten mit kryptogenem Schlaganfall der Verschluss eines offenen Foramen ovale in der Sekundärprävention des Schlaganfalls wirksam?

Hintergrund: Etwa 30-40\% aller gesunden Menschen haben ein offenes Foramen ovale, wobei die Inzidenz mit dem Alter abnimmt. Etwa $20 \%$ aller Schlaganfälle sind kryptogen, das heißt, es konnte keine kausale Ursache identifiziert werden. Eine Reihe von epidemiologischen Studien legten nahe, dass bei unter 60-jährigen Patienten mit kryptogenem Schlaganfall ein offenes Foramen ovale signifikant häufiger zu finden ist und dass diese Personen auch ein erhöhtes Rezidivrisiko für Schlaganfälle haben. Soweit beurteilbar, sind in dieser Patientengruppe Acetylsalicylsäure und eine orale Antikoagulation mit Vitamin-K-Antagonisten gleich wirksam.

In den Jahren 2000 bis 2010 wurden drei randomisierte Studien durchgeführt, in denen ein interventioneller Verschluss des offenen Foramen ovale bei Patienten mit kryptogenem Schlaganfall mit einer medikamentösen Therapie verglichen wurde. Die einzelnen Studien waren bezüglich des primären Endpunktes in der Regel erneuter Schlaganfall negativ, zeigten allerdings einen Trend zugunsten des Verschlusses des offenen Foramen ovale.

Kent DM, Dahabreh IJ, Ruthazer $\mathrm{R}$ et al. Device closure of patent foramen ovale after stroke: Pooled Analysis of Completed Randomized Trials. JACC 2016; 67: $907-17$
Patienten und Methodik: Es handelt sich um eine präspezifizierte Metaanalyse individueller Patientendaten von drei randomisierten Studien mit dem STARFLex der Firma NMT Medical und des
AMPLATZER PFO Occluder der Firma St. Jude Medical. Der primäre Outcome war Schlaganfall, TIA oder Tod. Der sekundäre Zielparameter war Schlaganfall. Die Analyse erfolgte mit einem Cox-Regressions-Modell, das die Intervention mit konservativer Therapie verglich.

Ergebnisse: Die Metaanalyse umfasste Daten von 909 Patienten der CLOSURE- [1], 414 der PC-Trial- [2] und 980 der RESPECTStudie [3]. Die Patienten waren im Mittel 45 Jahre alt. $30 \%$ litten unter einer Hypertonie. Etwa zwei Drittel hatten ein großes offenes Foramen ovale. Bei $90 \%$ lag ein Schlaganfall vor. Für den primären Endpunkt betrug die Ereignisrate pro Jahr 1,5\% in der Verschlussgruppe und 2,3\% in der konservativen Therapiegruppe. Das entspricht einer Hazard Ratio (HR) von 0,69, die mit einem p-Wert von 0,0517 nicht signifikant war. Bei einem CoxModell, das für kovariate Risiken adjustiert war, betrug die HR 0,68 und war mit einem p-Wert von 0,0491 signifikant. Für erneute ischämische Insulte betrug das Risiko $0,7 \%$ in der Verschlussgruppe und $1,3 \%$ in der konservativen Therapiegruppe, die $H R$ von 0,58 war mit einem p-Wert von 0,0407 signifikant. Bezüglich Untergruppen wie Alter, Geschlecht, Größe des offenen Foramen ovale, Behandlung mit Acetylsalicylsäure, TIA versus Schlaganfall, Migräne ja oder nein und oberflächliche versus tiefe Insulte in der Bildgebung ergaben sich keine Unterschiede.

Schlussfolgerungen: Bei Patienten mit offenem Foramen ovale und kryptogenem Schlaganfall führt der interventionelle Verschluss des offenen Foramen ovale mit einem starken Trend zu einer Reduktion des Endpunktes Schlaganfall, TIA und Tod. Dieses Ergebnis lässt sich jedoch nur erzielen, wenn für Kovariablen adjustiert wird.

\section{- Kommentar von Hans-Christoph Diener, Essen}

\section{Für uns bleiben die DGN-Leitlinien handlungsweisend}

Die unterschiedliche Betrachtungsweise der drei randomisierten Studien zum PFO-Verschluss bei kryptogenem Schlaganfall zwischen interventionellen Kardiologen und SchlaganfallNeurologen wird sich wahrscheinlich nie endgültig klären lassen, da es mit hoher Wahrscheinlichkeit keine weiteren Studien mit interventionellem PFO-Verschluss versus optimaler medikamentöser Therapie geben wird. Zudem wurden die Verschlusssysteme in der Zwischenzeit weiterentwickelt und sind bezüglich Verschluss- und Komplikationsrate verbessert worden. Gleichzeitig hat sich aber auch die Qualität der konservativen Therapie deutlich verbessert. Für den klinischen Alltag sind aber die Leitlinien der DGN weiterhin gültig, das heißt, dass bei Patienten mit kryptogenem Schlaganfall und PFO nur dann ein PFO-Verschluss erwogen werden sollte, wenn die $\mathrm{Pa}$ - tienten jung sind und wenn die Umstände der Schlaganfallentstehung eine paradoxe Embolie wahrscheinlich machen, wie das Auftreten des Schlaganfalls bei einem Valsalva-Manöver oder das Vorliegen einer tiefen Beinvenenthrombose. Leider ist völlig ungeklärt, wie vorzugehen ist, wenn ein Patient mit kryptogenem Schlaganfall und PFO unter konservativer Therapie ein Rezidiv erleidet. Das wurde in den randomisierten Studien nicht untersucht.

\footnotetext{
Referenzen:

1. Furlan AJ et al. N Engl J Med 2012; 366: 991 - 9

2. Meier B et al. N Engl J Med 2013; 368: $1083-9$

3. Carroll JD et al. N Engl J Med 2013; 368: 1092- 100
} 